\title{
Essentials for Best Practice: Treatment Approaches for Athletes With Eating Disorders
}

\author{
Jenny H. Conviser \\ Northwestern University \\ Amanda Schlitzer Tierney and Riley Nickols \\ The Victory Program at McCallum Place
}

\begin{abstract}
Eating disorders (EDs) and disordered-eating behaviors (DEBs), pose a high risk of morbidity and mortality, threatening physical health, emotional health, and overall quality of life. Unfortunately, among athletes, prevalence rates continue to increase. This document summarizes the challenges of establishing and navigating the multidisciplinary care needed to effectively treat EDs and DEBs among athletes. The benefits of timely and frequent communication within the multidisciplinary treatment team (MDTT) are emphasized and discussed. Authors advise who should be selected as members of the MDTT and suggest that all personnel, including athletic coaches, athletic trainers, physical therapists, and certified fitness professionals be ED-informed and ED-sensitive. Vital components of care are noted including use of a variety of evidence-based psychotherapeutic modalities, interventions which target emotional regulation, and prioritize values based compassionate care. Authors caution that performance decrements and medical/physiological changes are not always easily observable in individuals with EDs and DEBs and therefore, attuned, consistent, and ongoing monitoring is needed. Consensus regarding previously established parameters for return to play and careful titration of physical activity throughout the ED recovery process are suggested as important for preserving health, preventing re-injury, or relapse and facilitating successful return to sport participation.
\end{abstract}

Keywords: disordered-eating behaviors, athletes and eating disorders, ED treatment

All eating disorders (EDs) pose risk of psychological, social, and lifethreatening medical complications with serious compromise in overall quality of life (Styer, Conviser, Washburn, \& Aldridge, 2014). It is estimated that 10

Conviser is with Ascend Consultation in Health Care, LLC, Chicago, IL; and Northwestern University, Chicago, IL. Schlitzer Tierney and Nickols are with The Victory Program at McCallum Place, St. Louis, MO. Address author correspondence to Jenny H. Conviser at Jenny.Conviser@ASCENDchc.com. 
million women and 1 million men in the United States, of diverse gender, ethnicity, socio-cultural origin, socioeconomic status and age, will have EDs during their lifetime. However, there is greater prevalence of EDs among athletes than among non-athletes with rates varying from $6-45 \%$ in female athletes and $0-19 \%$ in male athletes (Glazer, 2008; Sundgot-Borgen \& Torstveit, 2004), with some rates of EDs among athletes appear to be increasing (Chatterton \& Petrie, 2013). Eating disorders are substantially different from more common eating-related and bodyrelated frustrations and, once established, do not remit without professional treatment. Timely access to care preserves health and minimizes deleterious effects of disordered-eating behaviors (DEBs) and EDs.

In this discussion, best practices for treatment of athletes with DEBs and EDs are summarized including the structure of a multidisciplinary treatment team (MDTT) and key components of ED treatment including: MDTT communication, evidence-based practices, weight and medical monitoring protocols, guidelines for navigating levels of treatment, optimizing the benefits of treatment, and strategies for relapse prevention.

\section{Multidisciplinary Treatment Team Model}

A multidisciplinary treatment team (MDTT) of professionals (see Figure 1), experienced in working with athletes and who have specialized training in EDs, is best positioned to provide well-coordinated, collaborative and effective care. The MDTT should consist of athletic personnel (i.e., coach, strength and conditioning coach, athletic trainer, medical director, etc.), physician, psychotherapist, registered dietician, psychiatrist, and professionals with other specialties. MDTT members must have experience in working with athletes and hold certification in sport and EDs in their respective field as available

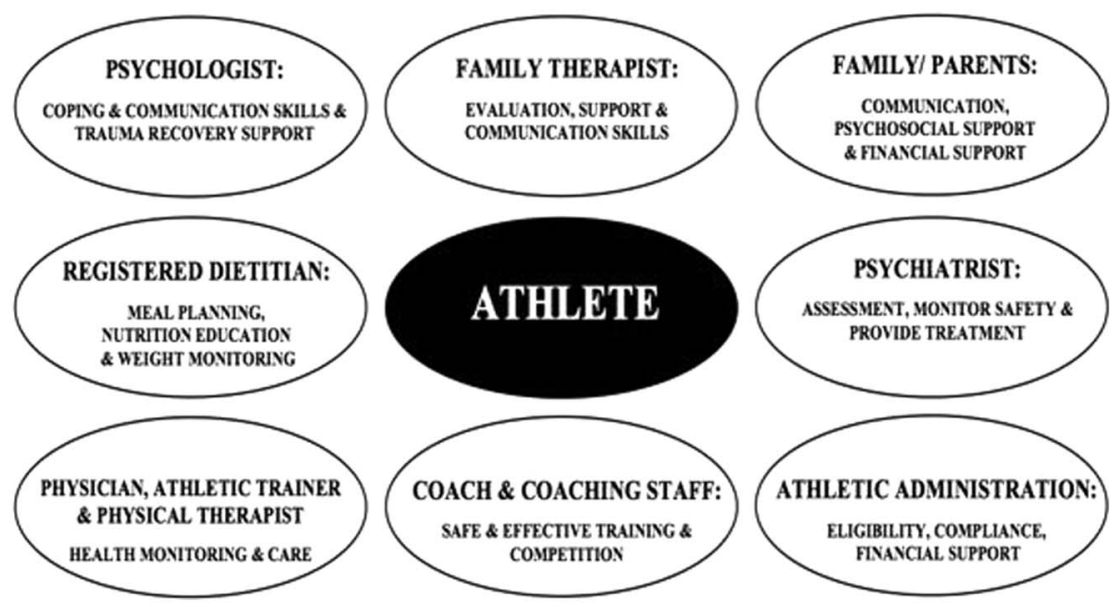

Figure 1 - Multidisciplinary, collaborative \& credentialed professional treatment providers, Conviser, Tierney, and Nickols, 2018. 
(e.g., Certified Mental Performance Consultant- CMPC; Certified Eating Disorder Specialist- CEDS; Certified Eating Disorders Registered Dietitian- CEDRD; or Board Certified in Sports Medicine- BCSM).

\section{Support Personnel}

Participation in ED treatment may require temporary modification of an athlete's schedule, training, competition, school, employment, and/or team obligations. It is recommended that athletic department personnel in academic settings, human resources staff in corporate locations, and support staff in professional environments, appropriately facilitate the athlete's treatment and recovery process (McArdle, Meade, \& Moore, 2016). The athlete's willingness to sign a Release of Information Form (ROI) gives all providers permission to share relevant information and helps create the foundation of good care. Constructive and timely communication throughout the course of treatment assists the MDTT and the athlete in responsible decision making regarding sport participation, treatment planning, finances, travel, housing, contracts, legal concerns, safety and health issues. Inability to obtain an ROI impairs treatment and recovery. If the athlete refuses to sign the ROI, the athlete's concerns should be explored, their questions answered, and referral to a more intensive level of care should be considered.

Athletes benefit from effective communication within the MDTT. However, psychological and medical health information is privacy protected according to federal law and must remain confidential. All MDTT members are expected to take reasonable precautions to protect confidentiality, and any limits of confidentiality need to be discussed at the outset of the relationship and updated as the process unfolds (American Psychological Association, 2017). The athlete must be informed of who will have access to their treatment information and in what format information will be communicated. The athlete should be informed that disclosure of information may occur 1) with legal permission, 2) when mandated by law, 3) when necessary to provide professional service, and 4) when necessary to protect the athlete or others from harm.

\section{Medical Care}

A physician, when available, may anchor and lead the MDTT. A physician conducts the initial medical exam, confers with the MDTT, monitors the athlete's well-being over time and refers the athlete to other medical specialists, as needed. The physician will evaluate, treat, and refer to other medical care providers and manage ED-related medical complications (see Figure 2) including cardiovascular, gastrointestinal, orthopedic, psychiatric and endocrinological conditions. Cooccurring conditions such as anxiety, depression, obsessive compulsive behavior, post-traumatic stress, substance use or abuse, and self-injury should be assessed, monitored and treated by available specialists (Jacobson \& Luik, 2014). Medical exams (see Figure 3) may include, but not be limited to laboratory tests, gowned weights, body composition, bone health analysis, sitting/standing blood pressure, resting heart rate, electrocardiogram, and growth chart documentation for children and adolescents. 


\section{GENERAL:}

-Marked weight loss, gain, or fluctuation

-Unexplained change in the growth curve in a child or adolescent

-Cold intolerance

-Weakness $\& /$ or Fatigue

-Presyncope \&/or Syncope

-Hot flashes, sweating episodes

ORAL AND DENTAL:

-Oral trauma/lacerations

-Perimyolysis

-Parotid gland enlargement

\section{NEUROPSYCHIATRIC:}

-Depressive/Anxious

- Obsersive/Compulsive behaviors

-Poor concentration \&/or Memory loss

-Insomnia

-Self-harm

-Suicidal thoughts, plans or attempts

-Seizures

ENDOCRINE:

-Amenorrhea or oligomenorrhea

-Low sex drive

-Stress fractures

-Low bone mineral density

-Infertility
CARDIORESPIRATORY:

-Chest pain

- Heart paptations

-Orthostatic tachycardia/hypotension

-Dyspnea

-Edema

GASTROINTESTINAL:

-Epigastr ic discomfort

-Abdominal bloating

-Early satiety

- Gastroesophageal reflux

-Hemaemesis

- Hemorrhoids \& rectal prolapse

-Constipation

DERMATOLOGIC:

- Lanugo har

- Hair loss

- Carotenoderma

-Russell's sign (calluses or scars

on the back of the hand)

-Poor wound healing

-Dry brittle hair and nails

Figure 2 - Signs and symptoms of eating disorders (Academy of Eating Disorders, 2016).

\section{Complete Blood Count:}

-Leukopenia, anemia, or thrombocytopenia

-Comprehensive metabolic panel to include electrolytes, renal function tests and liver enzymes, glucose, sodium, potassium, chloride, blood bicarbonate, blood urea nitrogen, creatinine, calcium, phosphate, magnesium, total protein, albumin, and prealbumin aspartate aminotransaminase (AST), alanine aminotransaminase (ALT)

-Electro-cardiogram (ECG), bradycardia, prolonged OTc $(>450 \mathrm{msec}),(E C G), \&$ other arrhythmias

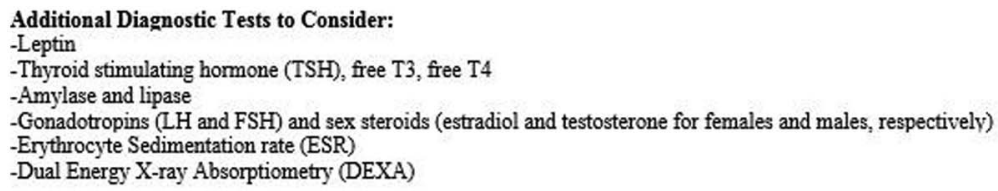

Figure 3 - Basic tests for medical evaluation (Academy of Eating Disorders, 2016). 
While the physician and medical personnel order medical tests and give clearance for physical activity specified via the parameters of frequency, intensity, mood and duration (FIMD), it is essential that all members of the MDTT assume responsibility for subsequent care and ensure the following: all ordered tests are completed, results are collected and interpreted, and the athlete is in compliance with all treatment recommendations. Input from the MDTT shall be considered in the initial evaluation, in ongoing re-evaluation and throughout the term of the athlete's team status or until the athlete is referred to another treatment team. It is beneficial to find consensus, clarity and consistency among members of the MDTT in communicating to the athlete any final decisions regarding health, treatment, physical activity and sport. Consensus regarding ED protocol is essential for good care and best treatment outcome.

Keep in mind that the athlete may not readily agree with treatment recommendations (Eisenberg, Golberstein, \& Gollust, 2007) and may avoid or delay compliance. This reluctance is a feature of the illness. If at any time, a member of the MDTT observes or believes often that the athlete is not following treatment and sport related recommendations, those observations should be immediately communicated to the MDTT. Sport training and participation should be suspended until the athlete completes re-evaluation and a revised treatment plan is drafted and agreed upon.

The MDTT may include a psychiatrist since EDs can influence or be influenced by mood, energy, and concentration (Markser, 2011). The psychiatrist's role includes: psychiatric evaluation, medication prescription, prescription management and monitoring emotional safety. It is beneficial for a psychiatrist to advise the appropriate level of ED treatment (see Table 1) and to contribute expertise concerning safety, medical care, diagnoses and sport participation.

\section{Mental Health Care Provider}

A licensed mental health care provider (MHCP) is a vital member of the MDTT. The MHCP should be a licensed psychologist, social worker, or counselor. The MHCP provides individual, group and family therapy, psychoeducation, and/or coping skills training. Unfortunately, not all counseling departments or professional sports organizations with in-house psychological support are able to provide the level of care or specificity of care needed to treat EDs. Psychotherapy for athletes with EDs requires specialty training and targets to improve interpersonal boundaries, communication, and emotional regulation. The MHCP assists in evaluating the athlete's mental status and readiness to return to sport participation.

\section{Evidence-Based Psychotherapy}

A variety of evidence-based psychotherapeutic modalities are utilized in best practice ED treatment plans. The primary targets of the therapeutic modalities will include improved emotion identification, expression and regulation. In addition, improved body image, normalized patterns of eating, balanced nutrition and restored body weight, as well as, family dynamics, community or team related stressors, relationship issues, sexuality, identity development, trauma history and other co-occurring conditions are often related to ED treatment targets that should be addressed. Evidence-based psychotherapies for EDs include: Cognitive Behavior Therapy (CBT), Enhanced Cognitive Therapy (CBT-E), Dialectical Behavior Therapy 


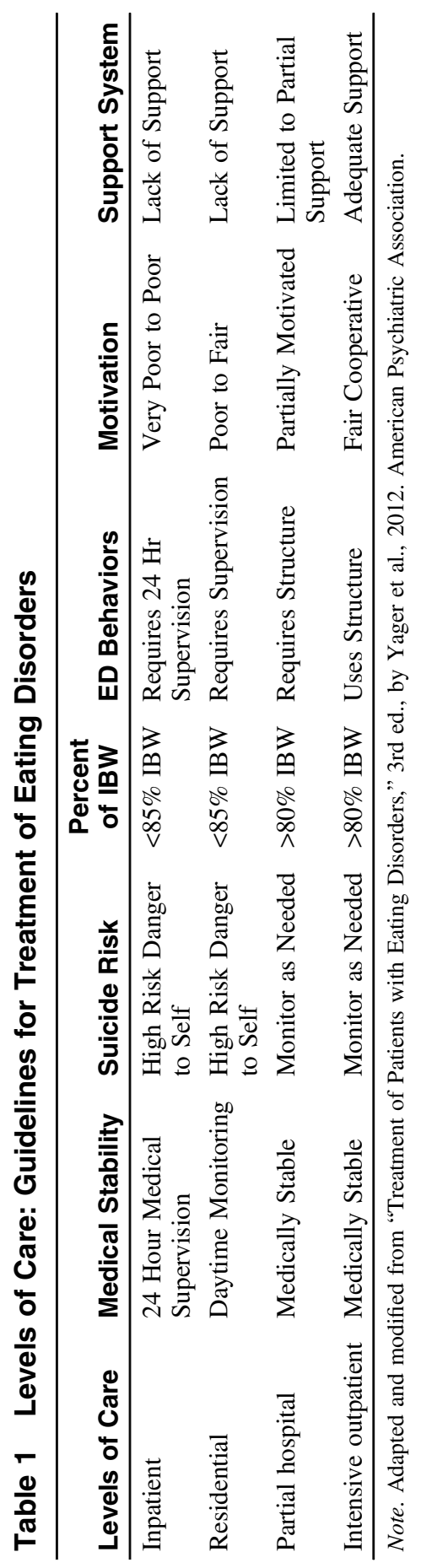


(DBT), Acceptance and Commitment Therapy (ACT), and Family-Based Treatment (domiciled children). Evidence-informed modalities include: Narrative Therapy, Eye Movement Desensitization and Reprocessing (EMDR), and experiential therapies such as yoga, meditation, and art, music, dance and movement therapy.

Behavioral therapies have the strongest empirical support for treating ED symptoms and related psychiatric disorders. Behavioral strategies used to combat ED pathology include: distress tolerance training, mindfulness, emotion regulation skills, behavioral modification, cognitive restructuring, healthy self-evaluation, and adaptive coping skills. Interventions are most effective when they empower change and best fit the athlete's self-identified needs and values (Touyz \& Hay, 2015) in the context of a well-coordinated, engaged and compassionate treatment team.

\section{Registered Dietitian}

The Registered Dietitian (RD) is a necessary member of the MDTT. The RD conducts nutrition assessment, provides sport nutrition education, drafts individualized meal plans, and supervises therapeutic meals. The RD understands the importance of balancing energy needs and energy expenditure to meet the demands of training, exercise, vital organ function and daily living. The RD guides and reinforces improved nutrition intake, assists in monitoring body weight and body composition, and conducts food exposures where indicated. The RD may be the first to notice evidence of decline in the athlete's functioning or signs of relapse as depicted by change in nutrition or body weight, body preoccupation, or food related rituals, perseveration, or rigidity.

Without ED specific interventions delivered by a MDTT, EDs are not likely to remit (Thompson, Petrie, \& Anderson, 2017). It is useful for all members of MDTT to be adaptive, to accommodate the athlete's evolving needs, and to establish and maintain parameters of care and ensure safety as they support ED recovery. In some instances, returning to sport is not aligned with an individual's health and recovery status and, as such, ongoing re-evaluation by the MDTT during treatment is necessary. It is critical that the MDTT agree upon previously established parameters for return to play (Cook et al., 2016) since a premature return to sport may increase risk of aggravated illness, injury or re-injury.

\section{Weight Monitoring}

Weight monitoring, often the responsibility of the RD, may be conducted by any member of the treatment team. Weigh-ins are completed on a variable schedule, while wearing gowns or with clothing status documented. In early phases of treatment, for the purpose of reducing anxiety, the athlete's back may be turned away from the scale while weighing ("blind weights") so weight values are obtained but not observable to the athlete. Goals for weight range and body composition must be individualized and consider: weight history (including highest and lowest body weights), athletic body type, sufficient hormonal functioning and maintaining a positive energy balance. When weighing, RDs should be aware of the potential for athletes to artificially add weight via water loading, weighted undergarments, weighted hair ties, and/or other means. Height must be reliably measured, and self-reported health information should be verified. Numerical information may 
aggravate EDs. Health care providers must consider potential risk and benefit when sharing information (i.e., body composition, weight, etc.).

The nature of certain sports may favor preferred physiques or body types and result in pressure to modify diet and body (Ackland, Maughan, Stewart, \& Muller, 2013). Dieting and focus on body weight change (upward or downward) are associated with greater ED risk, and therefore any performance related dietary advising should be communicated and guided by a sport certified dietitian (American Academy of Pediatrics, 2005; Rosendahl, Bormann, Aschenbrenner, Aschenbrenner, \& Strauss, 2009).

\section{Coach, Strength and Conditioning Coach, Athletic Trainer, \& Physical Therapist}

It is recommended that coaches, athletic trainers, physical therapists and certified fitness professionals, who are ED-informed or ED-certified, participate as active members of the MDTT. These professionals are well-positioned to detect psychological and physical changes associated with ED risk and to positively support recovery. All physical activity and training prescriptions must be accompanied by adequate nutritional support. Guidelines for physical activity will specify FIMD of training and competition (American College of Sports Medicine, 2018). The certified fitness professional, through collaborative communication and education of the MDTT, ensures that appropriate levels of training are prescribed and conducted. This will include periodization of training, guiding optimal biomechanical technique, encouraging adequate rest and recovery, and creating safe and supportive training environments. The ED-informed and ED-certified fitness professionals will thoughtfully anticipate physical and emotional challenges that could arise and assist the athlete in preparing to meet those challenges.

\section{Adjusting Physical Activity}

Athletic excellence typically requires an athlete to challenge their physical and psychological limits in order to meet the demands of a sport. Consistently performing at a high level requires dedication, a strong work ethic, perseverance, and coachability. At times, however, athletes may adopt the "more is better" mentality and develop patterns of overtraining that may contribute to physical and mental fatigue. DEBs and EDs and the associated chronic malnutrition impede improvement of strength, flexibility and aerobic conditioning. Overtraining, especially given the presence of or history of DEBs and EDs, diminished athletic performance, increased frustration, decreased confidence, and increases risk of exacerbated ED symptoms and behavior. In addition, the athlete may experience negative body awareness, appearance related preoccupation, hypervigilance, emotional dysregulation, and difficulty coping with the demands of sport, relationships and living (Petrie, Galli, Greenleaf, Reel, \& Carter, 2013). Body and appearance dissatisfaction may persist and/or intensify (Galli, Petrie, Reel, Chatterton, \& Baghurst, 2013).

While resuming physical training during or following treatment for a DEB or ED indicates significant progress, this phase of recovery is not without challenge especially given its frequent, gradual and tenuous nature. Athletes accustomed to 
extraordinary training loads may find that gradual and intentional training regimens at low levels of intensity feel relatively insufficient. Smaller training loads may prompt beliefs such as: "I am not doing enough", "I am not working hard enough" or "I am being lazy". The athlete may doubt their ED recovery capability and their ability to re-establish successful training and competition. Negative self-talk, fear and anxiety concerning their athletic future may ensue. Guilt specific to disappointing themselves and others may also emerge. Since EDs may be a way of coping with intense emotion, urges to use ED behaviors may thereby be aggravated (see Figure 4). All potential ED complications may therefore further intensify especially given a history of trauma, loss, anxiety or mood disorders.

Decisions regarding changes in frequency and intensity of sport activity and training must cautiously consider factors such as meal plan compliance and weight restoration status, vital signs, laboratory results, ED symptom status, emotion regulation capability, and compliance with all MDTT recommendations. Prescriptions for exercise and physical activity must be carefully adjusted throughout the process of

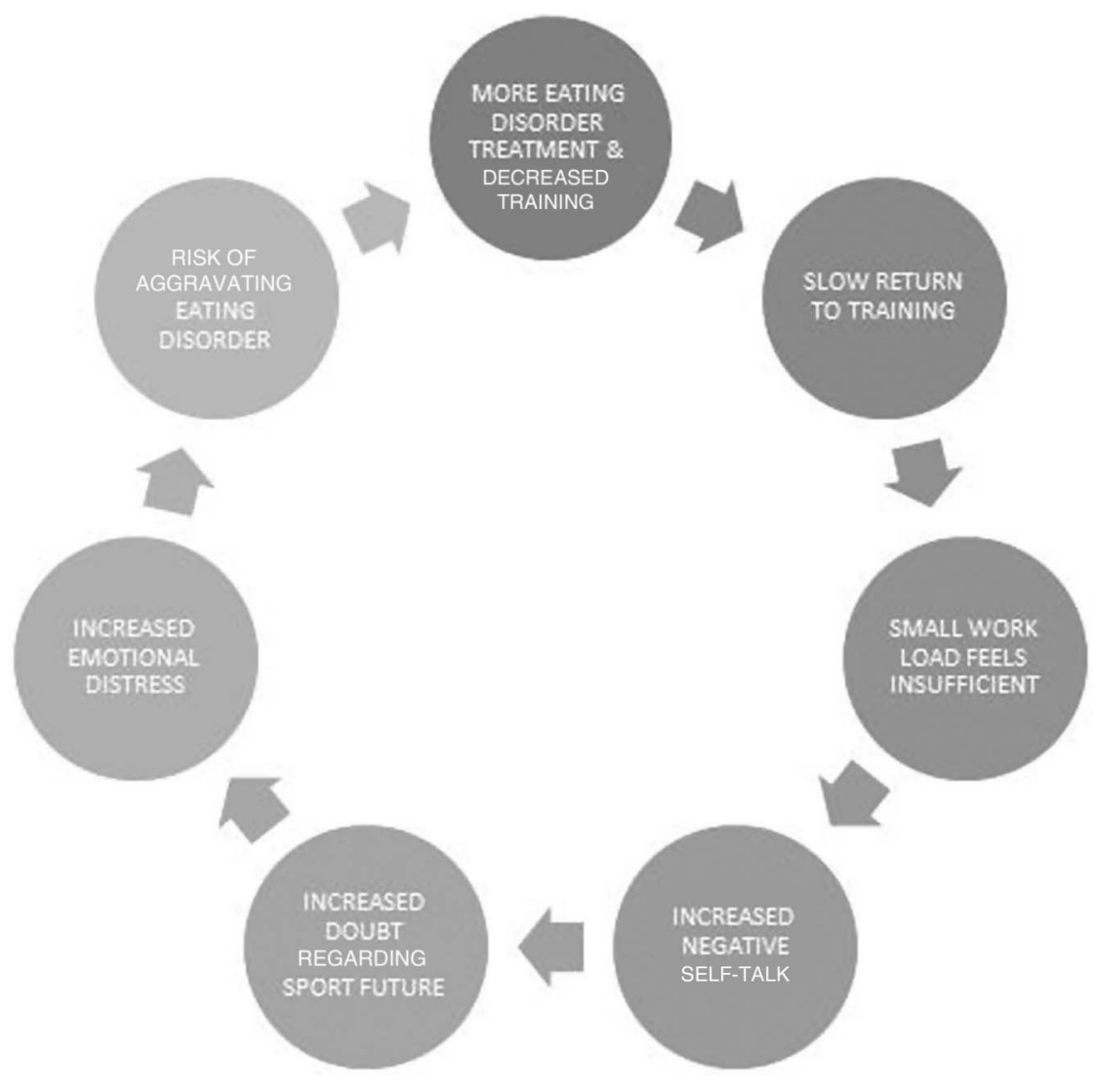

Figure 4 - Relapse Risk Among Athletes During Eating Disorder Recovery: Conviser, Tierney, and Nickols, 2018. 
treatment and recovery and must take into consideration all of the aforementioned factors. It is critical that the MDTT foster the athlete's healthy connection and awareness of body cues and appropriate related responses while recognizing that this often a slow and gradual recovery process which benefits from support by qualified professionals. The MDTT educates and supports athletes in optimal physical and mental training to improve performance and secure best overall health.

\section{Collaborative Communication}

Each member of MDTT should work within their scope of practice and relevant credentials and operate in close communication with each other. In an effort to best coordinate care and facilitate communication, MDTT members must provide their name, address, telephone, dates of service, type of service provided, relevant observations and recommendations for care for all other MDTT members. Any treatment contracts regarding safety, return to play parameters, guidelines for traveling with a team, medication compliance, etc., require universal agreement from the MDTT members and should be consistently enforced.

Since ED recovery occurs over a lengthy period of time and considering the signs of recovery are not always outwardly tangible, the athlete will depend on the MDTT and family members for patience and encouragement in order to remain compliant and hopeful. When collaborating within a MDTT, each MDTT member should assert their expert advice, even when it may not be supported by other members of the treatment team. The MDTT should make every effort to find consensus regarding treatment decisions and clinical recommendations, particularly when it may challenge the athlete's immediate training or team status.

\section{Transitioning Levels of Care}

Close collaboration with the athlete, treatment team, athletic personnel and the athlete's family is beneficial for successful return to training, competition, and independent living. Comprehensive documentation of health and treatment history is needed to establish guidelines for transitioning levels of care and to enhance communication to best inform an athlete's ongoing treatment. Expectations for academic, professional, and athletic participation, training load, and competition should be specified. The athlete and sport personnel must be familiar with early warning signs of potential lapse and understand the specific medical, psychological, and ED specific markers that warrant a higher level of care.

\section{Relapse Prevention Strategies}

Lapse or relapse may be common occurrences along a recovery path but are not indications of failure or inability to recover. As such, avoid any shaming or blaming an athlete who exhibits signs of a lapse or relapse. The MDTT can facilitate learning from lapses, sustain positive recovery and decrease the likelihood of a relapse by providing needed support. Physical, nutrition, sport performance, and emotional recovery must co-occur in synchrony. Action plans and treatment parameters should be agreed upon by all MDTT members, communicated to the athlete, and consistently upheld by all parties. 
The clinical signs and risks of relapse should be identified and made familiar to the athlete and all care providers prior to returning to athletics or transitioning to a lower level of care. Athletes should avoid rushing or abbreviating treatment as a premature return to training or competition during the ED recovery process as doing so has been shown to further increase the likelihood of a relapse (De Souza et al., 2014). Additionally, it is beneficial to draft pre-established and agreed upon plans for resumption of more intensive care. The athlete should be informed about what is entailed in intensive levels of care including the type and length of the treatments, location, treatment objectives, cost of treatment, and insurance coverage. All treatment providers should recognize when more intensive care (see Table 1) is needed and respond swiftly to access additional support according to the treatment plan.

Athletes will benefit from greater proficiency in accessing both internal resources (e.g., emotional coping skills, distress tolerance, self-awareness, and self-, etc.) and external sources of support (e.g., mental health care providers and services, academic assistance providers and services, nutrition support services and providers). Athletes may feel accustomed to managing emotional matters on their own and may avoid ED support because of related stigma and bias. Successful athletes in recovery will benefit from improved understanding of the value of utilizing all available sources of support (i.e., coach, medical team, athletic trainer, friends and family, and mental health professional).

\section{Clinical Treatment Implications}

The complexity and severity of EDs are underestimated, especially when the athlete is performing well, creating a false sense of health and wellness. In addition, athletes will characteristically work hard to meet the demands of the sport and mask any emerging performance deficiencies. Performance decrements and medical/physiological changes will not always be observable immediately following the onset of an ED. In fact, athletic performance can temporarily improve after the emergence of ED symptoms, which can motivate additional changes in weight or diet, and subsequently reinforce unhealthy and dangerous behaviors. The human body is resilient and able to function well for a finite period of time; however, ED complications resulting from prolonged states of energy deficiency and physical demands will eventually manifest with far-reaching implications for health and safety.

\section{Conclusion}

The sport environment has been shown to influence the risk of EDs, illness and premature death (Arcelus, Mitchell, Wales, \& Nielsen, 2011) but may also play a vital role in minimizing ED risk and fostering recovery (Coker-Cranney \& Reel, 2015). The aforementioned recommendations on the role of each MDTT member and considerations to support safe, appropriate treatment and training parameters, align with fostering an environment where the athlete is better able to navigate recovery and more consistently demonstrate an ability to adhere to treatment targets.

Unfortunately, the ED treatment and recovery process may be longer, more expensive, more time consuming, more trying and more convoluted than imagined or desired. There are many reasons to be frustrated and unsatisfied with change and 
growth that may appear relatively minor and intangible in the early phases of recovery. The ability to remain patient, hopeful, and persistent can be challenging, but all are vital to recovery. Commit to those treatment components that make positive differences such as respectful communication, safe and effective training guidelines, reassurance that gradual change is acceptable, focus on longer term goals, building trust and confidence in the treatment process and the MDTT, and choosing activities that are positively engaging (Plateau, Arcelus, McDermott, \& Meyer, 2015). Identify agreed upon parameters for treatment and return to play and avoid rushing to complete treatment or re-establish full training and competition. Prioritize the therapeutic alliance, permit unique individual differences to exist and avoid comparison with any standards other than himself/herself (Hay, Touyz, \& Sud, 2012). Targeting improvement of overall quality of life rather than symptom reduction alone has also been shown to be beneficial (Touyz \& Hay, 2015).

Confidence in the MDTT and in ED recovery are essential components of a successful recovery process. Athletes excel when teachers, coaches, sport personnel, family and friends believe in their ability to make progress, are not alarmed or angered by lapse or slowed progress and remain hopeful. Sport teams, athletic systems, health care providers, as well as families who leverage resources, awareness programming, policies and funding may collectively reduce risk of illness, relieve suffering, foster recovery and facilitate successful, safe and fulfilling return to sport participation.

\section{References}

Academy of Eating Disorders Report. (2016). Eating disorders: A guide to medical care (3rd ed.). Retrieved from https://www.aedweb.org/learn/publications/medical-care-standards

Ackland, T., Maughan, R., Stewart, A., \& Muller, W. (2013). How to minimize the health risks to athletes who compete in weight-sensitive sports. British Journal of Sports Medicine, 47, 1012-1022. doi:10.1136/bjsports-2013-092966

American Academy of Pediatrics [AAP]. (2005). Promotion of healthy weight-control practices in young athletes. Pediatrics, 116, 1557-1564. doi:10.1542/peds.2005-2314

American College of Sports Medicine. (2018). Guidelines for exercise testing and prescription (10th ed.). Philadelphia, PA: Wolters Kluwer.

American Psychological Association. (2017). Discussing limits of confidentiality, ethics code 4.02. Retrieved from https://www.apa.org/ethics/code/index.aspx

Arcelus, J., Mitchell, A.J., Wales, J., \& Nielsen, S. (2011). Mortality rates in patients with anorexia nervosa and other eating disorders. Archives of General Psychiatry, 68, 724-731. 21727255 doi:10.1001/archgenpsychiatry.2011.74

Chatterton, J.M., \& Petrie, T.A. (2013). Prevalence of disordered eating and pathogenic weight control behaviors among male collegiate athletes. Eating Disorders, 21, 328-341. 23767673 doi:10.1080/10640266.2013.797822

Coker-Cranney, A., \& Reel, J.J. (2015). Coach pressure and disordered eating in female collegiate athletes: Is the coach-athlete relationship a mediating factor? Journal of Clinical Sport Psychology, 9, 213-231. doi:10.1123/jcsp.2014-0052

Cook, B.J., Wonderlich, S.A., Mitchell, J.E., Thompson, R., Sherman, R., \& McCallum, K. (2016). Exercise in eating disorders treatment: Systematic review and proposal of guidelines. Medicine \& Science in Sports \& Exercise, 48, 1408-1414. 26909533 doi:10.1249/MSS.0000000000000912

De Souza, M.J., Nattiv, A., Joey, E., Misra, M., Williams, N., Mallinson, R.J., ... Matheson, G. (2014). Female athlete triad coalition consensus statement on 
treatment and return to play of female athlete triad: 1st International Conference held in San Francisco, California, May 2012 and 2nd International Conference held in Indianapolis, Indiana, May 2013. British Journal of Sports Medicine, 48, 289289. 24463911 doi:10.1136/bjsports-2013-093218

Eisenberg, D., Golberstein, E., \& Gollust, S.E. (2007). Help-seeking and access to mental health care in a university student population. Medical Care, 45, 594-601. 17571007 doi:10.1097/MLR.0b013e31803bb4c1

Galli, N., Petrie, T.A., Reel, J.J., Chatterton, J.M., \& Baghurst, T.M. (2013). Assessing the validity of the weight pressures in sport scale for male athletes. Psychology of Men \& Masculinity, 15, 170-180. doi:10.1037/a0031762

Glazer, J. (2008). Eating disorders among male athletes. Current Sports Medicine Reports, 7, 332-337. doi:10.1249/JSR.0b013e31818f03c5

Hay, P.J., Touyz, S., \& Sud, R. (2012). Treatment for severe and enduring anorexia nervosa: A review. Australian \& New Zealand Journal of Psychiatry, 46, 1136-1144. doi: $10.1177 / 0004867412450469$

Jacobson, C.M., \& Luik, C. (2014). Epidemiology and socio-cultural aspects of non-suicidal self- injury and eating disorders. In L. Claes\& J. Muehlenkamp (Eds.), Non-suicidal self-injury in eating disorders: Advancements in etiology and treatment (pp. 19-34). New York, NY: Springer Heidelberg. doi:10.1007/978-3-642-40107-7_2

Markser, V.Z. (2011). Sport psychiatry and psychotherapy. Mental strains and disorders in professional sports. Challenge and answer to societal changes. European Archives of Psychiatry and Clinical Neuroscience, 261, 182-185. 21901268 doi:10.1007/s00406011-0239-x

McArdle, S., Meade, M.M., \& Moore, P. (2016). Exploring attitudes toward eating disorders among elite athlete support personnel. Scandinavian Journal of Medicine \& Science in Sports, 26, 1117-1127. 26134346 doi:10.1111/sms.12515

Petrie, T., Galli, N., Greenleaf, C., Reel, J., \& Carter, J. (2013). Psychosocial correlates of bulimic symptomatology among male athletes. Journal of Psychology and Sport Excellence, 15, 680-687. doi:10.1016/j.psychsport.2013.09.002

Plateau, C.R., Arcelus, J., McDermott, H.J., \& Meyer, C. (2015). Responses of track and field coaches to athletes with eating problems. Scandinavian Journal of Medicine \& Science in Sport, 25, e240-e250. doi:10.1111/sms.12286

Rosendahl, J., Bormann, B., Aschenbrenner, K., Aschenbrenner, F., \& Strauss, B. (2009). Dieting and disordered eating in German high school athletes and non-athletes. Scandinavian Journal of Medicine Science and Sports, 19, 731-739. doi:10.1111/j. 1600-0838.2008.00821.x

Styer, D.M., Conviser, J.H., Washburn, J.J., \& Aldridge, D. (2014, March). Gender differences in quality of life and functional impairment associated with binge eating disorders: A clinical population study. Paper presented at the International Conference on Eating Disorders, New York City, NY.

Sundgot-Borgen, J., \& Torstveit, M.K. (2004). Prevalence of eating disorders in elite athletes is higher than in the general population. Clinical Journal of Sport Medicine, 14, 25-32. 14712163 doi:10.1097/00042752-200401000-00005

Thompson, A., Petrie, T., \& Anderson, C. (2017). Eating disorders and weight control behaviors change over a collegiate sport season. Journal of Science and Medicine in Sport, [Published online ahead of print]. http://www.sciencedirect.com/science/article/ pii/S1440244017303341

Touyz, S., \& Hay, P. (2015). Severe and enduring anorexia nervosa (SE-AN): In search of a new paradigm. Journal of Eating Disorders, 3, 26. 26236477 doi:10.1186/s40337015-0065-z 